\title{
Detrás de una circulación abdominal exuberante
}

\author{
Behind an exuberant abdominal circulation
}

A 54-year-old-man with a history of multifatorial lung disease (pulmonary obstructive lung disease GOLD IV group D and silicosis), presented at 2009 a right iliofemoral deep vein thrombosis (DVT). After then he had been hypocoagulated for 9 months. Five years later, presented a left iliofemoral deep vein thrombosis. Since then, is under uninterrupted hypocoagulation. An abdominal computed tomography performed at 2012 showed retroperitoneum and pelvic collateral circulation without evident thrombus at femoral level. At 2015 he was referred to a Hepatic Disease Consultation because of an exuberant abdominal venous collateral circulation with suspected underlying hepatic disease. Physical examination revealed an exuberant abdominal venous circulation (Figure 1) without palpable liver. The laboratory findings included negative ds-DNA and ANA; and positive beta-2 glycoprotein antibodies in two distinct determinations (12 weeks apart). A definitive diagnosis of antiphospholipid syndrome (APLS) was made. APLS is a systemic autoimmune disorder, which can occur as a primary condition or in the presence of another systemic disease, like systemic lupus erythematosus (in $40 \%$ of the cases). The diagnosis of APLS includes clinical and laboratory criteria. Clinical suspicion should be raised in presence of one or more otherwise unexplained venous or arterial thrombotic events, especially in young patients; one or more specific adverse outcomes related to pregnancy, including fetal death after 10 weeks gestation, premature birth due to severe preeclampsia or placental insufficiency, or multiple embryonic losses ( $<10$ weeks gestation), or other unexplained thrombocytopenia or prolongation of a test of blood coagulation. The typical laboratory finding is the presence of persistent antiphospholipid (aPL) antibodies. Anticoagulation (warfarin and other vitamin $\mathrm{K}$ antagonists - VKA, heparin) and/or antiplatelet drugs remains the mainstay of management in patients with thrombotic APLS. At present, direct oral anticoagulants should only be considered as an alternative, in patients with APLS and DVT, when there is a known VKA allergy or poor anticoagulant control. Indefinite anticoagulation for APLS is supported by evidence, since it carries a higher risk of recurrent thrombosis compared to aPL-negative subjects and the thrombotic risk may increase with time. The prognosis of patients with APLS is quite variable and depends on different factors (severity of primary clinical manifestation; past history of thrombosis; high levels of antibodies; adequate therapeutics, and presence of neoplasm).

\section{References}

1. Bustamante J and Bhimji. S. Antiphospholipid Syndrome. StatPearls Publishing; 29, April, 2017.

2. Arachchillage and Laffan M. Pathogenesis and management of antiphospholipid syndrome. British Journal of Haematology, 2017. doi: 10.1111/bjh.14632

3. Santamaria JR, Badziak D et al. Síndrome antifosfolípide. Anual Brasilian Dermatology 2005;80(3):225-39.
Figure 1, 2 and 3.

An exuberant abdominal venous circulation.
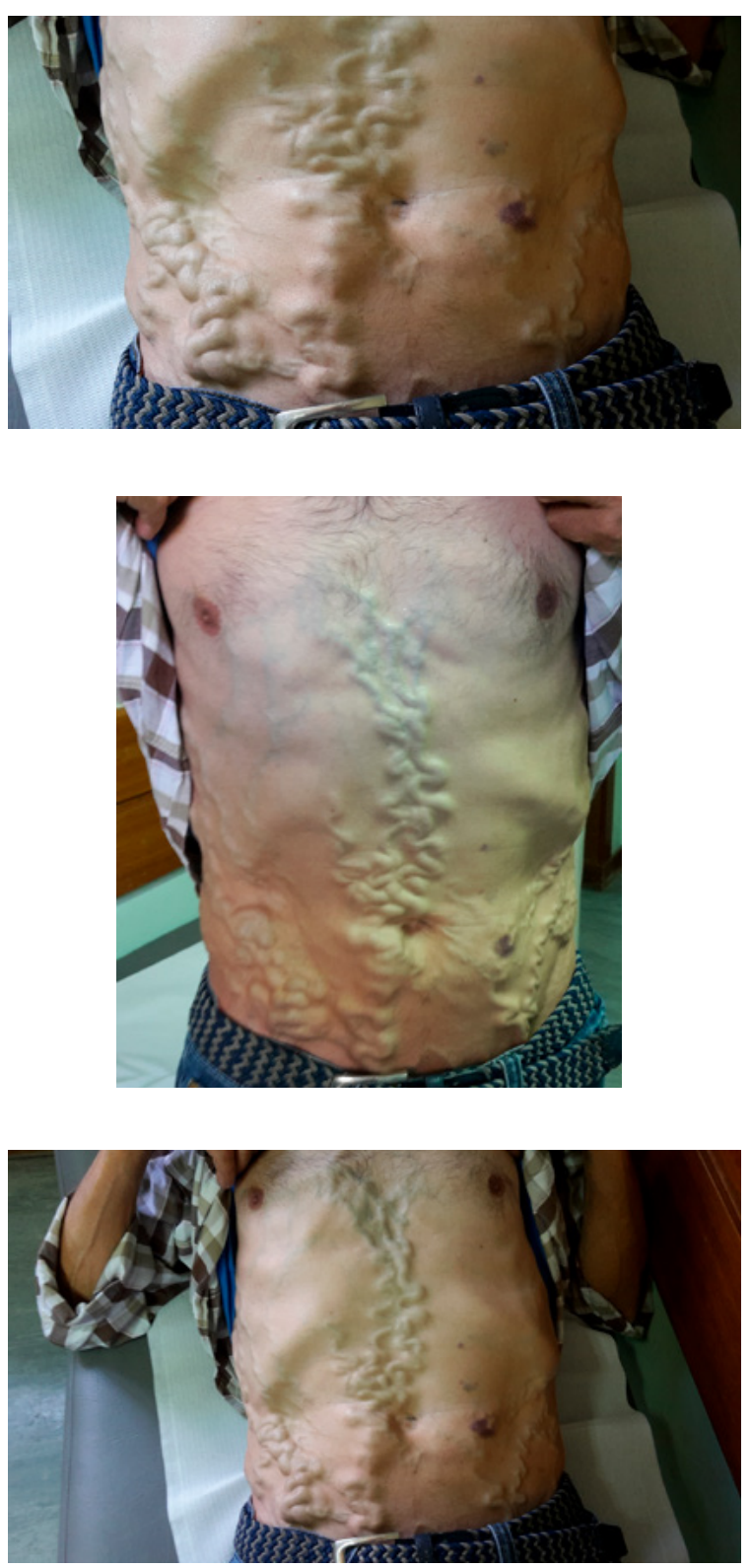

Diagnosis: Antiphospholipid syndrome (APLS)

\section{Diana Silva Fernandes, Marta Soares}

Department of Internal Medicine, Centro Hospitalar do Médio Ave. Famalicão, Portugal. 